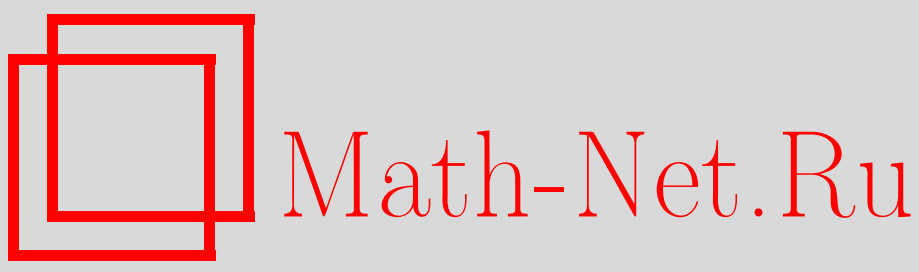

А. Р. Борисюк, Глобальные бифуркации на бутылке Клейна. Общий случай, $M a-$ тем. сб., 2005, том 196, номер 4, 3-22

DOI: https://doi.org/10.4213/sm1281

Использование Общероссийского математического портала Math-Net.Ru подразумевает, что вы прочитали и согласны с пользовательским соглашением

http://www.mathnet.ru/rus/agreement

Параметры загрузки:

IP: 54.164 .48 .24

26 апреля 2023 г., 15:44:29 
УДК 514.747

\author{
А.P. Борисюк
}

\title{
Глобальные бифуркации на бутылке Клейна. Общий случай
}

\begin{abstract}
В настоящей работе рассматривается однопараметрическое семейство гладких векторных полей в пространстве большой размерности такое, что при критическом значении параметра соответствующее поле обладает седлоузловым циклом. Рассматривается случай, когда гомоклинические орбиты этого цикла вместе с циклом образуют гладкую бутылку Клейна. Задача состоит в описании поведения множества траекторий при изменении параметра.

Библиографоя: 8 названий.
\end{abstract}

\section{§1. Введение}

1.1. Гомоклиническая бутылка Клейна седлоузлового цикла. Рассмотрим однопараметрическое семейство векторных полей в фазовом пространстве размерности не ниже четырех. Предположим, что нулевому (критическому) значению параметра в этом семействе соответствует седлоузловой цикл - периодическая орбита, один мультипликатор которой равен 1 , а остальные не лежат на единичной окружности. Такое вырождение неустранимым образом встречается в однопараметрических семействах. Предположим также, что гомоклинические траектории седлоузлового цикла заполняют гладкую поверхность, диффеоморфную бутылке Клейна. Это предположение не увеличивает коразмерности выражения.

При определенных условиях типичности, наложенных на семейство [1; § 5.5.3], в нем происходят следующие бифуркации. При малом отклонении параметра $\varepsilon$ в любую сторону от критического значения $\varepsilon=0$ гладкое инвариантное многообразие, диффеоморфное бутылке Клейна, сохраняется и гладко зависит от параметра. Эта теорема о сохранении (без утверж дения о гладкости по параметру) доказана для четырехмерного фазового пространства в [2], а для произвольной размерности в [3] и [2].

Инвариантная бутылка Клейна при нулевом значении параметра оказывается нормальным гиперболическим многообразием в смысле [4]. Теорема о сохранении и гладкости для таких многообразий доказана Н. Феничелем [5]. На ней и основано современное доказательство сохранения гомоклинической бутылки Клейна [3]. Теорема Феничеля утверждает больше. А именно, нормальная гиперболичность сохраняется при малых возмушениях. Кроме того, в малой окрестности притягиваюшего нормального гиперболического многообразия каждая траектория не

Работа вьполнена при финансовой поддержке Российского фонда фундаментальных исследований (грант № 02-01-00482) и CRDF (грант № RM1-2358-MO-02). 
только приближается к этому многообразию, но и “выбирает” траекторию на инвариантном многообразии и к ней экспоненциально стремится. Таким образом, с точки зрения численного эксперимента каждая точка, близкая к инвариантному многообразию, за конечное время выходит на это многообразие, а затем движется по одной из траекторий ограничения системы на это многообразие. Тем самым для описания поведения системы вблизи многообразия достаточно изучить систему на этом многообразии. Поэтому ниже рассматриваются только семейства уравнений на бутылке Клейна. Критическому значению параметра соответствует седлоузловой цикл, гомоклинические траектории которого заполняют всю бутылку. При отклонении параметра $\varepsilon$ в одну сторону от критического значения (скажем, влево) седлоузловой цикл распадается на два грубых: устойчивый и неустойчивый; при отклонении в другую сторону (скажем, вправо) - исчезает. При этом и происходят наиболее интересные бифуркации; они исследуются ниже.

При возвращении параметра справа к критическому значению $\varepsilon=0$ происходит “катастрофа голубого неба", открытая впервые В. С. Медведевым [6]. Малому положительному значению параметра соответствуют два предельных цикла, которые удлиняются при стремлении параметра к нулю и в пределе исчезают (см. $[1 ; \S 5.5]$ и п. 1.1.1 ниже). Кроме того, происходит бесконечное число бифуркаций удвоения, а также рождения и исчезновения предельного цикла. Эти бифуркации так же, как и их “периодический” характер, обнаружены в [1]. Слово “периодический" поставлено в кавычки, так как периодичность бифуркаций в зависимости от параметра наблюдается лишь приблизительно и только после замены параметра: $a=T(\varepsilon), T \rightarrow \infty$ при $\varepsilon \rightarrow 0^{+}$. Цель настоящей работы - полностью описать последовательность бифуркаций за период.

Оказывается, что по уравнению, соответствующему критическому значению параметра $\varepsilon=0(a=\infty)$, можно построить конечньй набор функций одного переменного, взаимное расположение максимумов и минимумов которых полностью определяет последовательность происходящих в семействе бифуркаций. Другими словами, по критическому уравнению можно описать полный бифуркационный сценарий, реализующийся в семействах общего положения на гомоклинической бутылке Клейна.

Этому описанию и посвящена настоящая работа. Теорема 1 позволяет построить бифуркационный сценарий по данному семейству. Теоремы 2 и 3 описывают множество всех бифуркационных сценариев, которые при этом построении возникают.

1.1.1. Глобальное отображение Пуанкаре. В работе [7] показано, что исследование глобальных бифуркаций в семействе гомоклинических бутылок Клейна может быть сведено к изучению семейства меняющих ориентацию диффеоморфизмов окружности (глобальное отображение Пуанкаре). Итак, рассмотрим семейство

$$
\widetilde{f}_{a}: x \mapsto-x+a+h_{a}(x)
$$

где $x \in \mathbb{S}^{1}$, а семейство функций $h_{a}$ сходится к некоторой функции $h_{0}$ при $a \rightarrow \infty$ в норме пространства $C^{3}$. Отображение $(1)$ - диффеоморфизм, если и только если

$$
h_{a}^{\prime}(x)<1
$$


В $[7]$ исследованы бифуркации этого семейства в случае, когда функция $h_{0}$ (а следовательно, функция $h_{a}(x)$ для любого достаточно большого $a$ ) унимодальная (морсовская с одним минимумом и одним максимумом на своем периоде). В этой статье мы рассмотрим семейство (1) с произвольной функцией $h_{a}$, удовлетворяюшей требованиям типичности, которые будут сформулированы ниже.

Приведем два элементарных утверждения, доказательства которых можно найти в $[1 ; \S 5.5]$.

ПРЕДЛОЖЕНИЕ 1. Меняющий ориентацию диффеоморфизм окружности имеет ровно две неподвижные точки.

ПРЕДЛОЖЕНИЕ 2. Отображение окружности степени (-1) не имеет ииклов периода больие двух.

В системе (1) встречаются два типа бифуркаций: при изменении параметра неподвижная точка может поменять свою устойчивость, при этом рождается или исчезает 2-цикл (происходит бифуркация удвоения периода или обратная к ней); бифуркация седлоузловой периодической орбиты периода 2: исчезновение (рождение) циклов периода 2 через слияние двух циклов.

Настояшая работа преследует две цели. Во-первых, построить бифуркационный сценарий по заданному семейству функций $h_{a}$. Во-вторых, описать все возможные бифуркационные сценарии для произвольной функции $h_{a}$ с фиксированным числом экстремумов. Поставленная задача сначала решена для частного случая отображения (1), когда $h_{a} \equiv h$ :

$$
f_{a}: x \mapsto-x+a+h(x),
$$

а затем показано, что результаты остаются верными и в случае семейства (1).

1.2. Основные предположения. В этом пункте мы сформулируем требования общности положения на функции $h_{a}$, рассматриваемые ниже.

1) При любом фиксированном значении параметра $a$ функция $h_{a} 2 \pi$-периодическая.

2) При любом фиксированном значении параметра $a$ функция $h_{a}$ морсовская c $n+1$ экстремумом на своем периоде $[0 ; 2 \pi)$. В силу предположения 1 $n$ - нечетное число.

Покажем, что семейство функций $h_{a}$ может быть нормировано. Далее все рассуждения проводятся для нормированного семейства.

ПРЕДЛОЖЕНИЕ 3. Рассмотрим семейство диффеоморфизмов окружности (1) с функциями $h_{a}$, стремящимися $x$ функиии $h$ по норме пространства $C^{3}$ при $a \rightarrow \infty$. Тогда поворотом окружсности на угол $\xi(a)$, стремящийся $\kappa$ пределу при а $\rightarrow \infty$, и заменой параметра а семейство (1) можно преобразовать в семейство аналогичного вида с функииями $h_{a}$, удовлетворяющими следующим условиям: $h_{a}(0)=h_{a}(2 \pi)=0 ; h_{a}^{\prime}(0)=h_{a}^{\prime}(2 \pi)=0 ; h_{a}(x)>0$ при $x \in(0 ; 2 \pi)$. 
ДокАЗАТЕЛЬСТво. Пусть $\xi(a) \in[0 ; 2 \pi)$ - абсцисса глобального минимума функции $h_{a}(x)$, а $\eta(a)$ - ордината.

Сделаем замену координат $y=x-\xi(a)$. Отображение примет вид:

$$
\begin{aligned}
& f_{a}: y+\xi(a) \mapsto-y-\xi(a)+a+h_{a}(y+\xi(a)), \\
& f_{a}: y \mapsto-y+(a-2 \xi(a)+\eta(a))+h_{a}(y+\xi(a))-\eta(a),
\end{aligned}
$$

где $y \in[0 ; 2 \pi]$. Пусть $\widetilde{a}=a-2 \xi(a)+\eta(a), \widetilde{h}_{\widetilde{a}}(y)=h_{a}(y+\xi(a))-\eta(a)$, тогда $f_{\widetilde{a}}: y \mapsto-y+\widetilde{a}+\widetilde{h}_{\widetilde{a}}(y)$, причем в этих координатах семейство отображений $f_{\widetilde{a}}$ будет удовлетворять условиям предложения.

Глобальный минимум $\xi(a)$ функции $h_{a}$ удовлетворяет уравнению $h_{a}^{\prime}=0$. Поскольку функция $h$ морсовская, ее производная в окрестности каждого своего нуля обратима. Кроме того, никакие два критические значения функции $h$ не совпадают. Поэтому ее глобальный минимум гладко зависит от параметра $a$. Следовательно, зависимость $\widetilde{h}_{\widetilde{a}}$ от параметра $\widetilde{a}$ является $C^{3}$-гладкой по построению. Предложение полностью доказано.

Для того чтобы сформулировать последние два требования, введем ряд определений.

ОПРЕДЕЛЕНИЕ 1. Рассмотрим функцию $h$, удовлетворяющую условию (2), требованиям типичности 1$), 2$ ) и условиям нормировки. Определим разбиение $\Omega=$ $\left\{\omega_{i}\right\}$ отрезка $[0 ; 2 \pi]$, порожденное функцией $h$ так, что точки разбиения - прообразы критических значений функции $h$.

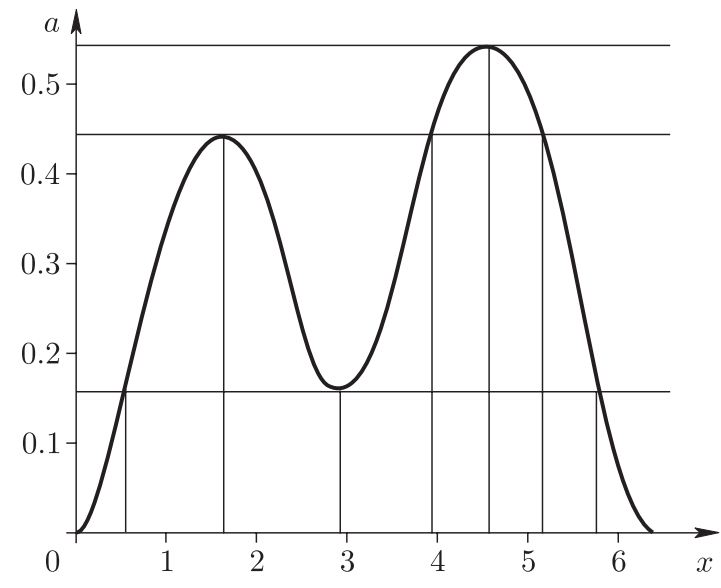

Рис. 1. Построение разбиения $\Omega$

Данное определение проиллюстрировано на рис. 1. Приведенная функция $h$ будет использована ниже для иллюстрации основных утверждений.

В п. 2.2 будет доказано следующее предложение. 
ПРЕДЛОЖЕНИЕ 4. Число отрезков разбиения $\Omega$, пороххденного нормированной функиией $h$ с п әкстремумами на интервале $(0 ; 2 \pi)$ (n нечетно), может принимать любое значение из множества

$$
A(n)=\left\{3 n-1+4 k \mid k=0,1, \ldots, \frac{(n-1)(n-3)}{8}\right\} .
$$

ОПРЕДЕЛЕНИЕ 2. Два отрезка разбиения $\Omega$ называются эквивалентными, если их $h$-образы совпадают.

Определение 2 задает отношение эквивалентности и, следовательно, разбивает множество отрезков разбиения $\Omega$ на классы эквивалентности.

ОПРЕДЕЛЕНИЕ 3. Базисныц отрезком в классе әквивалентности отрезков разбиения назовем левый (с наименьшими координатами концов) отрезок данного класса.

ОПРЕДЕЛЕНИЕ 4. Любым двум эквивалентньм отрезкам разбиения $\omega_{k}$ и $\omega_{j}$ поставим в соответствие функции $i_{k j}$ и $i_{j k}$ по следуюшему правилу: $i_{k j}: \omega_{k} \rightarrow \omega_{j}$ так, что $h(x)=h\left(i_{k j}(x)\right)$, функция $i_{j k}$ строится аналогично на отрезке $\omega_{j}$. Функции $i_{k j}$ для всех возможных номеров $k, j$ назовем инволютивньими функииями.

Определение мотивировано тем, что $\omega_{k} \stackrel{i_{k j}}{\longrightarrow} \omega_{j} \stackrel{i_{j k}}{\longrightarrow} \omega_{k}$, причем $i_{j k}\left(i_{k j}(x)\right)=x$, $x \in \omega_{k}$.

ОПРЕДЕЛЕНИЕ 5 . Функции $p_{k j}(x)$, принадлежащие набору

$$
\left\{a=p_{k j}(x)=x+i_{k j}(x)-h(x), x \in \omega_{k}\right\},
$$

называются функциями ииклов семейства (3).

Теперь мы можем сформулировать условия типичности, которые налагаются на функцию $h$, задающую семейство (3) (в дополнение к сформулированньм выше предположениям 1), 2)).

3) Функция циклов $p_{k j}(x), x \in \omega_{k}$, для любых номеров $k$ и $j$ является морсовской.

4) Любые две функции циклов $p_{k_{1} k_{2}}, p_{k_{3} k_{4}}$ (за исключением случая, когда $k_{1}=k_{4}, k_{2}=k_{3}$ ) не имеют одинаковых критических значений.

ЗАмечание. Справедливо следуюшее: $p_{j k}=p_{k j} \circ i_{j k}$.

1.3. Бифуркационный сценарий. Для описания полного бифуркационного сценария надо задать все пары точек $(x, a)$, где $x$ - точка 2-цикла семейства $f_{a}: x \mapsto-x+a+h(x)$ при значении параметра $a$. Мы предполагаем, что функция $h$ нормированная и удовлетворяет требованиям типичности 1)-4) из п. 1.2.

ПреДЛОЖенИЕ 5. Рассмотрим семейство (3) и разбиение отрезка $[0 ; 2 \pi]$, порожденное функиией $h$. Пусть $\left\{i_{k j}\right\}$ - набор инволютивных функиий, где номера $(k, j)$ пробегают номера всех пар әквивалентных отрезков. Рассмотрим набор функиий $\left\{a=p_{k j}(x)=x+i_{k j}(x)-h(x), x \in \omega_{k}\right\}$. Множество точек $(x, a)$, принадлежащих графикам функиий набора $\left\{p_{k j}\right\}$, совпадает с множеством пар $(x, a)$, әде $x$ - точка 2 -иикла семейства $f_{a}$. 
ТЕОРема 1. В семействе (3) с функиией $h$, удовлетворяющей требованиям типичности, с возрастанием параметра а происходят следующие бифуркаиии (см. рис. 2).

1) При прохождении параметра через локальный минимум (максимум) одной из функиий ииклов набора $\left\{p_{k j}\right\}$, не соответствующий критическому значению функции $h$, происходит рождение (исчезновение) двух периодических орбит периода 2: одна устойчивая, другая неустойчивая.

2) При значениях параметра, равных $a_{j}=2 x_{j}-h\left(x_{j}\right)$, где $x_{j}-$ точка локального әкстремума функиии $h$, происходит бифуркация удвоения периода (при прохождении параметра через локальный минимум одной из функиий ииклов) или бифуркация, обратная к бифуркации удвоения периода (при прохождении через локальный максимум).

Доказательства этих утверждений приведены в п. 2.2.

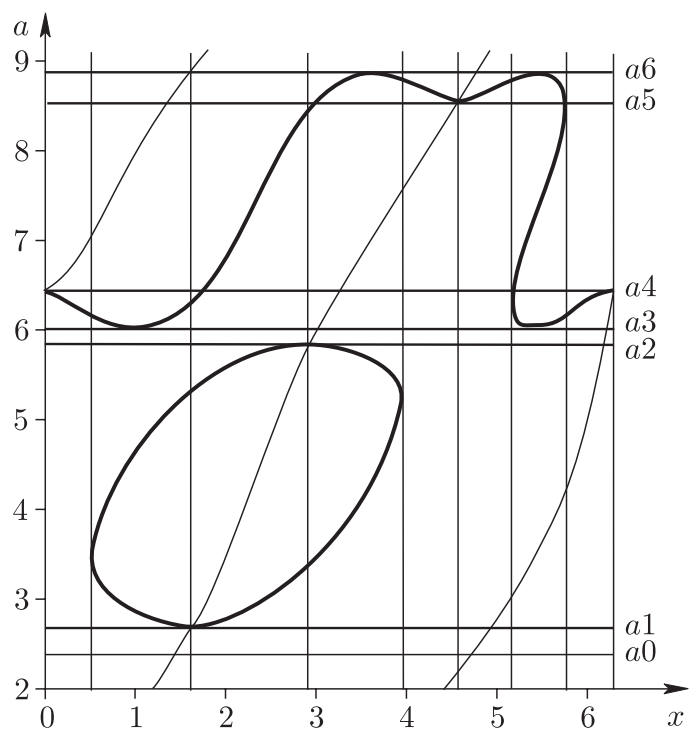

Рис. 2. Бифуркационный сценарий

ПояснЕнИя К РИс. 2. Ось абсцисс разбита на отрезки разбиения $\Omega$, как это было показано ранее на рис. 1 . На каждом отрезке разбиения определены функции циклов. Опишем картину при фиксированных значениях параметра $a$.

1) Значение параметра $a=$ “ $a 0$ ”. Согласно предложению 1 система имеет ровно две неподвижные точки при каждом значении параметра. Линия $a=$ “ $a 0$ ” пересекает линии, соответствующие линиям неподвижных точек, и не пересекает функции циклов, а следовательно, система не имеет циклов периода два при этом значении параметра.

2) Значение параметра $a=$ “ $a 1$ ”. При этом значении левая неподвижная точка меняет устойчивость и рождается цикл периода два (случай 2) в теореме 1). 
3) Значение параметра $a=$ " $a 2$ ". Неподвижная точка меняет свою устойчивость, сливаясь с циклом периода два.

4) Значение параметра $a=$ " $a 3$ ". Это значение является бифуркационньм происходит рождение двух циклов периода два (случай 1) в теореме 1).

5) Значение параметра $a=$ " $a 4$ ". Неподвижная точка меняет свою устойчивость, сливаясь с циклом периода два.

6) Значение параметра $a=$ " $a 5$ ". При этом значении левая неподвижная точка меняет устойчивость и рождается цикл периода два (случай 2) в теореме 1).

7) Значение параметра $a=$ “ $a 6$ ". Устойчивый и неустойчивый циклы периода два сливаются и исчезают.

ПРЕДЛОЖЕНИЕ 6. Зафиксируем отрезок разбиения $\omega_{l}$. Пусть $p_{l l_{1}}, \ldots, p_{l l_{m}}$ - функции циклов, определеннье на $\omega_{l}$. Тогда

$$
\max _{j=l_{1}, \ldots, l_{m}} \max _{x \in \omega_{l}} p_{l j}(x)-\min _{j=l_{1}, \ldots, l_{m}} \min _{x \in \omega_{l}} p_{l j}(x)<2 \pi .
$$

Доказательство этого утверж дения можно найти в п. 2.5 .

ЗАмечАнИЕ. Из предложения 6 следует, что последовательность бифуркаций повторяется с периодом $2 \pi$, поэтому семейство (3) естественно рассматривать при значениях параметра $a \in[0 ; 2 \pi]$.

1.4. Реализация бифуркационных сценариев. Из теоремы 1 следует, что бифуркационный сценарий полностью задается набором функций циклов $\left\{p_{k j}\right\}$. В п. 2.3 будет доказано, что для того, чтобы задать набор $\left\{p_{k j}\right\}$, достаточно задать функции циклов на базисных отрезках.

ОПРЕДЕЛЕНИЕ 6. Характеристический граф-ориентированный граф на нумерованных вершинах, удовлетворяющий следующим свойствам: существуют такая нормированная функция $h$, удовлетворяющая условиям типичности, и соответствующее ей разбиение $\Omega$ (см. определение 1 ), что можно установить взаимно однозначное соответствие между вершинами графа и отрезками разбиения $\Omega$; каждая вершина, соответствующая базисному отрезку (такие вершины будем называть базисныцми), соединена направленньми ребрами со всеми вершинами, которые соответствуют отрезкам того же класса эквивалентности.

ОПРЕДЕЛЕниЕ 7. Ориентированный граф̆ на нумерованных вершинах называется квазихарактеристическим порядка $n$, если он удовлетворяет следующим условиям.

1) Вершины бывают двух типов:

а) из вершины выходит нечетное число ребер в вершины со старшими номерами;

b) в вершину входит ровно одно ребро.

2) Количество вершин первого типа (они называются базиснымми) равно $n$, а общее число вершин принадлежит множеству $A(n)$ (см. предложение 4).

Заметим, что характеристический граф функции $h$ является квазихарактеристическим порядка $n$, где $n$ - число экстремумов нормированной функции $h$ на интервале $(0 ; 2 \pi)$. 
НАПОМИНАНИЕ [8]. Updown подстановка - подстановка, у которой каждый элемент либо меньше двух соседних, либо больше соседних.

Из определения 6 видно, что updown подстановка функции $h$ однозначно определяет характеристический граф̆ набора функций циклов для соответствующего семейства диффеоморфизмов окружности (3). В п. 2.3 будет доказано обратное утверждение, а именно:

ПРЕДЛОЖЕНИЕ 7. Пусть задан характеристический граф функиии $h$, удовлетворяющей требованиям типичности. Тогда ирdошп подстановка функции однозначно восстанавливается по графу.

ТЕОРемА 2. Существует алгоритм, который определяет является ли квазихарактеристический граф характеристическим.

Описание алгоритма из теоремы 2 приведено в п. 2.4. Характеристический граф функции $h$ однозначно задает нумерацию функций циклов семейства (3), порожденных данной функцией $h$ и определенных на базисных отрезках. А именно, ребру граффа, соединяюшему вершины $k$ и $j$, соответствует функция циклов $p_{k j}$ на базисном отрезке $\omega_{k}$. Следуюшее предложение описьвает свойства функций циклов $p_{k j}$ на базисных отрезках.

ПРЕДЛОЖЕНИЕ 8. Рассмотрим базисный отрезок $\omega_{k}$ и функиии ииклов $p_{k k_{1}}, \ldots, p_{k k_{m}}$, определенные на этом отрезке. Функции ииклов $p_{k k_{l}}$ удовлетворяют следующим условиям.

1) Пусть $\omega_{k} \leqslant \omega_{k_{1}} \leqslant \cdots \leqslant \omega_{k_{m}}-\kappa л а с с$ әквивалентных отрезков, тогда для любого $x \in \omega_{k}$

$$
p_{k k_{1}}(x) \leqslant p_{k k_{2}}(x) \leqslant \cdots \leqslant p_{k k_{m}}(x)
$$

причем равенство мохет быть только в граничных точках отрез$\kappa a \omega_{k}$.

2) Если $j=k_{1}, k_{3}, \ldots, k_{m}$, то для любого $x \in \omega_{k}$ выполнено $p_{k j}^{\prime}(x)<1$. Если $j=k_{2}, k_{4}, \ldots, k_{m-1}$, то для любого $x \in \omega_{k}$ вицолнено $p_{k j}^{\prime}(x)>0$.

3) Если $j=k_{2}, k_{4}, \ldots, k_{m-1}$, то для любого $x \in \omega_{k}$

$$
p_{k j}^{\prime}(x)>\max _{l=k_{1}, k_{3}, \ldots, k_{m}} p_{k l}^{\prime}(x) .
$$

4) Существуют такие числа $a_{k}, 0<a_{k}<x_{k}$, что в граничных точках базисного отрезка выполнены следующие равенства:

$$
\begin{aligned}
p_{k k_{1}}\left(x_{k-1}\right)-x_{k_{1}} & =p_{k k_{2}}\left(x_{k-1}\right)-x_{k_{2}-1}=\cdots=p_{k k_{m-1}}\left(x_{k-1}\right)-x_{k_{m-1}-1} \\
& =p_{k k_{m}}\left(x_{k-1}\right)-x_{k_{m}}=x_{k-1}-a_{k-1}, \\
p_{k k_{1}}\left(x_{k}\right)-x_{k_{1}-1} & =p_{k k_{2}}\left(x_{k}\right)-x_{k_{2}}=\cdots=p_{k k_{m-1}}\left(x_{k}\right)-x_{k_{m-1}} \\
& =p_{k k_{m}}\left(x_{k}\right)-x_{k_{m}-1}=x_{k}-a_{k} .
\end{aligned}
$$

Доказательство этого предложения приведено в п. 2.3. Следующая теорема показывает, что любой набор функций, удовлетворяющий условиям предложения 8, реализуется как набор функций циклов для некоторого $h$. 
Теорема 3. Зафиксируем характеристический граф. Рассмотрим произвольное разбиение отрезка $[0 ; 2 \pi]$ такое, что количество отрезков разбиения равно числу вериин графа. Пусть отрезок разбиения $\omega_{k}$ соответствует базисной вершине. $Н а \omega_{k}$ заданы функции $p_{k j}$, где $j$ пробегает номера всех вершин графа, в которые ведут ребра из $k$-й вершины. Пусть, кроме того, функции $p_{k j}$ удовлетворяют ограничениям, наложсенным на функиии ииклов предложением 8. Тогда существует морсовская функиия $h$, удовлетворяющая требованиям типичности, такая, ито набор функиий ииклов соответствующего семейства (3) совпадает с заданньм набором функиий $p_{k j}$.

Теперь остается заметить, что теоремы 2,3 решают проблему реализации бифуркационных сценариев в семействах вида (3).

1.5. Эквивалентность бифуркационных сценариев. Введем понятие эквивалентности бифуркационных сценариев для семейств вида (3).

ОПРЕДЕЛЕНИЕ 8. Бифуркационные сценарии семейств отображений $F_{a}: x \mapsto$ $-x+a+f(x)$ и $G_{a}(x): x \mapsto-x+a+g(x)$ локально әквивалентны, если характеристические графы для функций $f(x)$ и $g(x)$ одинаковы и, кроме того, для любой фиксированной пары номеров $(k, j)$ соответствующие функции циклов лево-право эквивалентны.

Из этого определения следует, что для локально эквивалентных сценариев экстремумы функций циклов можно поставить во взаимно однозначное соответствие и, следовательно, правомерно следующее определение.

ОПРЕДЕЛЕниЕ 9. Два локально эквивалентных сценария называются эквивалентныцми, если выполнено следуюшее условие. Пусть функции циклов $p_{k j}, p_{m s}$ одного из сценариев имеют экстремумы в точках $x_{1}$ и $x_{2}$ соответственно, кроме того, $p_{k j}\left(x_{1}\right)>p_{m s}\left(x_{2}\right)$. Тогда соответствуюшие экстремумы другого сценария находятся в таком же соотношении.

Это определение мотивировано тем, что эквивалентные бифуркационные сценарии представляют собой одинаковые последовательности бифуркаций.

\section{§2. Бифуркационный сценарий}

2.1. Неподвижные точки. Приведем доказательство предложения 1 в несколько расширенной форме.

ПРЕДЛОЖЕНИЕ 9. Меняющий ориентацию диффеоморфизм окружности имеет ровно две неподвижные точки. Неподвижные точки семейства (3) и только они задаются равенством $a=\alpha(x)=2 x-h(x)$. Мультипликатор $\mu_{x_{0}}$ отображения $f_{a}$ в неподвижной точке $x_{0}$ по модулю меньше единицы тогда и только тогда, когда $h^{\prime}\left(x_{0}\right)>0$.

ДокАЗАтЕльство. Рассмотрим диффеоморфизм $f_{a}$. Пусть $\widehat{f}_{a}-$ накрываюшее отображение:

$$
\widehat{f}_{a}(x+2 \pi)=\widehat{f}_{a}(x)-2 \pi, \quad \widehat{f}_{a}^{\prime}(x)<0 .
$$

Без ограничения общности $\widehat{f}_{a}(0)=q \in[0 ; 2 \pi)$. Пусть $x_{0}$ - неподвижная точка $f_{a}$, тогда $\widehat{f}_{a}(x)=x(\bmod 2 \pi)$. График $\widehat{f}_{a}(x)=\tau$ один раз пересекает каждую из 
прямых $l_{0}: \tau=x, l_{-1}: \tau=x-2 \pi$ и никогда не пересекает линии $l_{n}: \tau=x+2 \pi n$ при $n \neq 0,-1$. Следовательно, $f_{a}$ имеет ровно две неподвижные точки.

Неподвижные точки отображения $f_{a}$ задаются уравнением $f_{a}(x)=x$, которое записываетсяв виде $-x+a+h(x)=x$. Отсюда получаем, что $a=\alpha(x)=2 x-h(x)$.

Далее, пусть $f_{a}(x)=x$. Имеем $f_{a}^{\prime}(x)=-1+h^{\prime}(x)$. Из условия (2) следует, что $f_{a}^{\prime}<0$. Поэтому условия $\left|f_{a}^{\prime}(x)\right|<1$ и $h^{\prime}(x)>0$ равносильны.

2.2. Периодические траектории. Приведем некоторые очевидные свойства разбиения $\Omega$, порож денного функцией $h$, удовлетворяющей требованиям типичности.

ПРЕДЛОЖЕНИЕ 10. 1) Функиия $h$ монотонна на любом отрезке разбиения. 2) Возможны три варианта взаимного расположения $h$-образов отрезков разбиения $\Omega$ : не пересекаются, имеют одну общую граничную точку, совпадают.

ДокаЗАтЕльство. 1) Пусть при некотором $i_{0} \exists x, \widetilde{x} \in \omega_{i_{0}}: h(x)=h(\widetilde{x})$, но в силу непрерывности функции $h$ это означает, что меж ду $x$ и $\widetilde{x}$ лежит локальный экстремум функции $h$, а это невозможно, так как точки локальных экстремумов точки разбиения. Пришли к противоречию.

2) Пусть $h$-образы отрезков $\omega_{j}$ и $\omega_{k}$ пересекаются во внутренней точке, т.е. для некоторого $x_{0} \in \omega_{j}$ найдется $\widetilde{x}_{0} \in \omega_{k}$ такое, что $h\left(x_{0}\right)=h\left(\widetilde{x}_{0}\right)$. Покажем, что в этом случае $h$-образы отрезков $\omega_{j}, \omega_{k}$ совпадают. Предположим противное: найдется $x \in \omega_{i}$, для которого не существует $\widetilde{x} \in \omega_{k}$ такого, что $h(x)=h(\widetilde{x})$. Но тогда в силу непрерывности функции $h$ на $[0 ; 2 \pi]$ и монотонности этой функции на $\omega_{j}$ и $\omega_{k}$ получим, что существует $\widehat{x} \in \omega_{j}$ такое, что $h(\widehat{x})=h\left(x^{m}\right)$, где $x^{m}-$ локальный экстремум функции $h(x)$. Пришли к противоречию с определением разбиения $\Omega$. Утверждение полностью доказано.

ДОКАЗАТЕЛЬСТВО ПРЕДЛОЖЕНИЯ 4. СогЛасно опреДеЛению 1 точками разбиения являются прообразы критических значений функции $h$. Следовательно, количество точек разбиения зависит от взаимного расположения локальных экстремумов функции $h$.

1) Прежде всего отметим, что точки локальных экстремумов ( $n$ штук) и концы отрезка $[0 ; 2 \pi]$ являются точками разбиения.

2) Найдем минимальное число точек разбиения $\Omega-\widetilde{A}_{\min }$, полученных при пересечении прямых $y=x^{j}$ ( $x^{j}$ - локальные экстремумы $\left.h(x)\right)$ с графиком функции $y=h(x)$. Каждый экстремум (кроме глобального максимума) дает как минимум две точки разбиения. Следовательно, число точек разбиения принимает минимальное значение в том и только том случае, когда все экстремумы дают ровно две точки разбиения. Итак, $\widetilde{A}_{\min }=2(n-1)$.

3) Произвольная функция $h$ может быть получена из функции $h_{\min }$, дающей минимальное число точек разбиения, конечным числом повторов следующей процедуры. Изменим один из локальных минимумов так, чтобы он стал менњше одного из локальных максимумов. При этом число точек разбиения увеличится на 4 . Так как единственное, что влияет на число точек разбиения, - взаимное расположение локальных экстремумов по оси $O y$, то число точек разбиения может принимать любое значение вида $\widetilde{A}=\widetilde{A}_{\min }+4 k$ до некоторого $\widetilde{A}_{\max }$. 


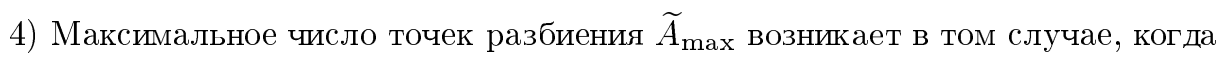
все локальные минимумы функции $h$ меньше ее локальных максимумов. Первый (с наименьшей $y$-координатой) локальный минимум дает две точки разбиения, а каждый последующий на две точки больше; первый локальньй максимум дает столько же точек разбиения, сколько и последний минимум, а каждый последующий на две менњше. Следовательно,

$$
\widetilde{A}_{\max }=\left(2+2 \cdot 2+2 \cdot 3+\cdots+2 \cdot\left(\frac{n-1}{2}\right)\right) \cdot 2=\frac{n^{2}-1}{2} .
$$

Итак, число точек разбиения может принимать любое значение из множества

$$
\widetilde{A}=\left\{n+2+\widetilde{A}_{\min }+4 k \mid k=0,1, \ldots,\left(\frac{\widetilde{A}_{\max }-\widetilde{A}_{\min }}{4}\right)\right\}
$$

а число отрезков разбиения

$$
A=\left\{n+1+\widetilde{A}_{\min }+4 k \mid k=0,1, \ldots,\left(\frac{\widetilde{A}_{\max }-\widetilde{A}_{\min }}{4}\right)\right\}
$$

Предложение 4 доказано.

Теперь все готово для доказательства основных утверждений.

ДоКАЗАТЕЛЬСТво ПРЕДЛОЖЕНИЯ 5. Уравнение 2-цикла: $f_{a}\left(f_{a}(x)\right)=x$. Имеем

$$
f_{a}\left(f_{a}(x)\right)=x-a-h(x)+a+h\left(f_{a}(x)\right)=x-h(x)+h\left(f_{a}(x)\right)=x .
$$

Получаем $h(x)=h\left(f_{a}(x)\right)$. Нас интересует случай $x \neq f_{a}(x)$, т.е. $x$ не является неподвижной точкой. Из предложения 10 следует, что уравнение 2-циклов имеет решение тогда и только тогда, когда $x \in \omega_{k}, f_{a}(x) \in \omega_{j}$, где $\omega_{k}$ и $\omega_{j}$ - эквивалентные отрезки. Тогда в силу определения 4 получаем, что $f_{a}(x)=i_{k j}(x)$. Пусть теперь $(k, j)$ - всевозможные пары номеров эквивалентных отрезков разбиения $\Omega$. Итак, мы будем иметь 2-цикл $\left(x, i_{k j}(x)\right), x \in \omega_{k}$, при значении параметра $a=p_{k j}(x)$, где $p_{k j}(x)=x+i_{k j}(x)-h(x)$. Предложение 5 доказано.

ПРЕДЛОЖЕНИЕ 11. Пусть $\omega_{k} u \omega_{j}$ - произвольные әквивалентные отрезки разбиения $\Omega$, причем отрезок $\omega_{k}$ лежит левее отрезка $\omega_{j}$. Рассмотрим $x \in \omega_{k}$, тогда 2-иикл $\left(x, i_{k j}(x)\right)$ семейства $f_{a}$ имеет мультипликатор $\mu_{x}$ такой, что

$$
\left|\mu_{x}\right|<1 \Leftrightarrow h^{\prime}\left(i_{k j}(x)\right) p_{k j}^{\prime}(x)<0, \quad\left|\mu_{x}\right|>1 \Leftrightarrow h^{\prime}\left(i_{k j}(x)\right) p_{k j}^{\prime}(x)>0
$$

где $p_{k j}(x)$ - функция ииклов семейства $f_{a}$, соответствующая әквивалентнылм отрезкам $\omega_{k}, \omega_{j}$. 
ДоказАтельство. Рассмотрим цикл $\left(x, i_{k j}(x)\right), x \in \omega_{k}$. Данньй 2-цикл устойчив тогда и только тогда, когда

$$
\left|\left(f_{a}\left(f_{a}(x)\right)\right)^{\prime}\right|<1, \quad\left(f_{a}\left(f_{a}(x)\right)\right)^{\prime}=\left(1-h^{\prime}\left(i_{k j}(x)\right)\right)\left(1-h^{\prime}(x)\right), \quad x \in \omega_{k},
$$

но в силу предположения (2) $\left(f_{a}\left(f_{a}(x)\right)\right)^{\prime}>0 \forall x \in \omega_{k}$, поэтому исходное неравенство сводится к неравенству $\left(f_{a}\left(f_{a}(x)\right)\right)^{\prime}<1$. Из равенства $h\left(i_{k j}(x)\right)=h(x)$ имеем $h^{\prime}\left(i_{k j}(x)\right) i_{k j}^{\prime}(x)=h^{\prime}(x)$. Подставим это в выражение (7) и получим

$$
\begin{gathered}
-h^{\prime}\left(i_{k j}(x)\right)-h^{\prime}\left(i_{k j}(x)\right) i_{k j}^{\prime}(x)+h^{2}\left(i_{k j}(x)\right) i_{k j}^{\prime}(x)<0 \\
-h^{\prime}\left(i_{k j}(x)\right)\left(1+i_{k j}^{\prime}(x)-h^{\prime}\left(i_{k j}(x)\right) i_{k j}^{\prime}(x)\right)<0 \\
-h^{\prime}\left(i_{k j}(x)\right)\left(1+i_{k j}^{\prime}(x)-h^{\prime}(x)\right)<0 .
\end{gathered}
$$

Кроме того, мы знаем, что

$$
p_{k j}^{\prime}(x)=1+i_{k j}^{\prime}(x)-h^{\prime}(x) .
$$

Теперь утверждение предложения 11 немедленно следует из выписанных соотношений.

ДокАЗАТЕЛЬСТво ТЕОРЕМЫ 1. Пусть

$$
a_{\min }=\min _{(k, j)}\left\{\min _{x \in \omega_{k}} p_{k j}(x)\right\}, \quad a_{\max }=\max _{(k, j)}\left\{\max _{x \in \omega_{k}} p_{k j}(x)\right\} .
$$

Рассмотрим семейство прямых $y=a$, где $a$ изменяется от $a_{\min }$ до $a_{\max }$. Пусть прямая $y=a$ пересекает графики набора функций циклов $p_{k j}(x)$ над отрезком $[0 ; 2 \pi]$ в $2 m$ точках с абсциссами $x^{1}, \ldots, x^{2 m} ;$ точки $x^{j}$ некритические для функций циклов. Число точек пересечения будет четное, так как $p_{j k}\left(i_{k j}(x)\right)=p_{k j}(x)$, $x \in \omega_{k}$, т.е. если прямая $y=a$ пересекает графии функции $p_{k j}$ в точке $x \in \omega_{k}$, то эта прямая пересекает и график функции $p_{j k}$ в точке $i_{k j}(x) \in \omega_{j}$. Тогда согласно предложению 5 семейство $f_{a}$ имеет $m$ 2-циклов $\left(x^{l}, i_{k j}\left(x^{l}\right)\right), k<j$, при значении параметра $a$.

Докажем утверждения теоремы в случае прохож дения параметра через локальный минимум одной из функций циклов. Утверждения для локальных максимумов доказываются аналогично.

1) Пусть $\widetilde{a}$ - невырожденный минимум функции циклов $p_{k j}(x), x \in \omega_{k}$. Пусть прямая $y=\widetilde{a}-\varepsilon$ при некотором малом $\varepsilon$ пересекает график функции $p_{k j}(x)$ в $q$ точках, тогда в силу морсовости функции $p_{k j}$ для $y=\widetilde{a}+\varepsilon$ точек пересечения будет $q+2$, т.е. рождаются два 2 -цикла. В силу предположения типичности 4 ) количество точек пересечения прямой $y=a$ с остальньми графиками функций циклов при прохождении $a$ через $\widetilde{a}$ не изменится.

2) Пусть $x_{j+1}$ - точка локального экстремума функции $h$. Тогда отрезки разбиения $\omega_{j}$ и $\omega_{j+1}$ эквивалентны и возникает функция циклов $p_{j j+1}$ такая, что $p_{j j+1}\left(x_{j+1}\right)=2 x_{j+1}-h\left(x_{j+1}\right)$. Тем самым мы получили утверждение 2$)$ теоремы.

Перейдем к доказательству теорем 2 и 3 , которые вместе составляют теорему о реализации. 


\section{3. Восстановление updown подстановки по ее характеристическо-} му графу.

ПреДЛОЖенИЕ 12. Рассмотрим разбиение $\Omega$, соответствующее функuии h. Рассмотрим класс эквивалентных отрезков этого разбиения. Тогда функция $h$ и функции циклов на небазисных отрезках этого класса однозначно восстанавливаются по значениям функции $h$ и функций циклов на базисном отрезке этого класса.

ДокАЗАТЕЛЬСтво. Пусть $\omega_{k}$ - базисный отрезок рассматриваемого класса, $\omega_{j_{1}}$ и $\omega_{j_{2}}-$ два других отрезка того же класса. По функции $\left.h\right|_{\omega_{k}}$ и функциям циклов $p_{k j_{1}}, p_{k j_{2}}$ восстанавливаются инволютивные функции $i_{k j_{1,2}}=p_{k j_{1,2}}+x+h(x)$. По этим данньм восстанавливаются функции $\left.h\right|_{\omega_{j_{1,2}}}$ и $p_{j_{1} j_{2}}$ :

$$
\begin{gathered}
\left.h\right|_{\omega_{j_{1,2}}}=\left.h\right|_{\omega_{k}} \circ i_{j_{1,2} k}, \\
i_{j_{1} j_{2}}=i_{k j_{2}} \circ i_{j_{1} k} .
\end{gathered}
$$

Напомним, что $i_{j k}=i_{k j}^{-1}$. Следовательно, $p_{j_{1} j_{2}}=i_{j_{1} j_{2}}-x-\left.h\right|_{\omega_{j_{1}}}$. Предложение 12 доказано.

ЗАмечАниЕ. На каждом базисном отрезке определено столько функций циклов, сколько сушествует отрезков, эквивалентных данному базисному, причем число этих функций нечетно.

Опишем сначала соответствие меж ду свойствами функции $h$ и ее характеристического графа.

1) Обшая вершина двух соседних эквивалентных отрезков разбиения является экстремумом функции $h$.

2) Максимумы и минимумы нормированной морсовской функции $h$ чередуются; самый левый ненулевой экстремум на интервале $(0 ; 2 \pi)$ - максимум.

3) Занумеруем классы эквивалентных отрезков разбиения $\Omega$ в порядке появления соответствующих базисных отрезков при проходе периода $[0 ; 2 \pi)$ слева направо. Если общая точка двух соседних эквивалентных отрезков разбиения - максимум, то она отображается функцией $h$ в правую вершину базисного отрезка из того же класса эквивалентности; если - минимум, то в левую. Тем самьм номер класса эквивалентности экстремума характеризует значение функции $h$ в нем, и его можно интерпретировать как "номер класса эквивалентности по вертикали".

Теперь все готово для доказательства предложения 7. Проиллюстрируем доказательство этого предложения на примере, обсуждавшемся выше при доказательстве теоремы 1.

ДОКАЗАТЕЛЬСТВО ПРЕДЛОЖЕНИЯ 7. Рассмотрим характеристический граф̆ функции $h$ с нумерованньми вершинами (см. пример на рис. 3 для функции $h$, приведенной на рис. 1). Двум соседним эквивалентным (т.е. соединенным с одной базисной) вершинам соответствует критическая точка функции $h$. Занумеруем эти точки по возрастанию номеров соответствуюших пар вершин графа. Самая левая 
ненулевая из этих пар точек - максимум функции $h$; дальше минимумы и максимумы чередуются.

Занумеруем базисные вершины граффа числами от 1 до $n$ в порядке возрастания их номеров как вершин графа. Припишем номер базисной вершины всем эквивалентньм, т.е. соединенным с ней ребрами, вершинам. Каждому максимуму сопоставим номер класса эквивалентности соответствующих соседних эквивалентных вершин графра. Каж дому минимуму сопоставим аналогичный номер, уменьшенньй на 1. Таким образом, $j$-му экстремуму функции $h$ отвечает номер соответствующей базисной вершины для максимумов и тот же номер, уменьшенный на 1 , для минимумов. Полученная подстановка $\{1, \ldots, n\} \rightarrow\{1, \ldots, n\}$ обозначается $\delta$. Подстановка $\{0,1, \ldots, n\} \rightarrow\{0,1, \ldots, n\}, 0 \mapsto 0, j \mapsto \delta(j), j \neq 0$, и является updown подстановкой функции $h$.

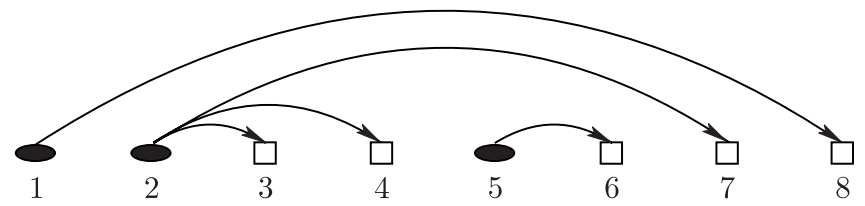

Рис. 3. Характеристический графф: $\bigcirc-$ базисные вершины, $\square-$ небазисные вершины

В рассматриваемом примере парам вершин $(2,3)$ и $(5,6)$ соответствуют максимумы функции $h$, а паре $(3,4)$ соответствует локальный минимум. Подстановка выглядит следующим образом: $\delta:(1,2,3) \mapsto(2,1,3)$, a updown подстановка $(0,2,1,3)$.

\section{4. Распознавание характеристических графов.}

ДокАЗАТЕЛЬСтво тЕоРЕмЫ 2. Рассмотрим квазихарактеристический граф с $n$ базисными вершинами и установим, является ли он характеристическим графом. Для этого применим алгоритм из предыдушего пункта.

1) Занумеруем пары соседних эквивалентных вершин характеристического графа в порядке возрастания их номеров. Назовем эти пары квазиэкстремумами.

2) Самую левую из этих пар назовем квазимаксимумом. Дальше квазиэкстремумы разбиваются на чередуюшиеся квазиминимумы и квазимаксимумы.

3) Занумеруем базисные вершины квазихарактеристического графа в порядке возрастания их номеров в множестве всех вершин графа. Полученный номер вершины назовем "вертикальнымм". Если число базисных вершин не совпадает с числом квазиэкстремумов, алгоритм останавливается. Здесь и далее это означает, что квазихарактеристический граф̆ не является характеристическим.

4) Если число базисных вершин совпадает с числом квазиэкстремумов, припишем каждому квазиэкстремуму “вертикальный” номер по следуюшему правилу. Этот номер равен “вертикальному” номеру соответствующей базисной вершины для квазимаксимума и на единицу меньше этого номера для 
квазиминимума. Обозначим через $\delta$ полученное отображение множества $\{1, \ldots, n\}$ в себя. Если отображение $\sigma:\{0,1, \ldots, n\} \rightarrow\{0,1, \ldots, n\}, 0 \mapsto 0$, $j \mapsto \delta(j), j \neq 0$, не является updown подстановкой, алгоритм останавливается.

5) В противном случае берется морсовская функция на окружности с построенной updown подстановкой. Затем строится ее характеристический граф. Если он не совпадает с исходным, алгоритм останавливается. В противном случае исходньй граф является характеристическим графом.

Описание алгоритма на этом закончено, и теорема 2 тем самьм доказана.

\section{5. Свойства функций циклов.}

ДоКАЗАТЕЛЬСТво ПРЕДЛОЖЕнИЯ 6. Предположим, что утверждение неверно, т.е. найдутся такой отрезок разбиения $\omega_{\beta}$ и такая точка $\xi \in \omega_{\beta}$, что

$$
p_{\beta \beta_{1}}(\xi)+2 \pi=p_{\beta \beta_{m}}(\xi) .
$$

Это равенство можно расписать следующим образом:

$$
\xi+i_{\beta \beta_{1}}(\xi)-h(\xi)+2 \pi=\xi+i_{\beta \beta_{m}}(\xi)-h(\xi),
$$

откуда мы получаем, что должно быть выполнено $i_{\beta \beta_{1}}(\xi)+2 \pi=i_{\beta \beta_{m}}(\xi)$. Однако мы знаем, что $i_{\beta \beta_{1}}(\xi) \in \omega_{\beta_{1}}, i_{\beta \beta_{m}}(\xi) \in \omega_{\beta_{m}}$, следовательно, $i_{\beta \beta_{m}}(\xi) \leqslant 2 \pi$, $i_{\beta \beta_{1}}(\xi)+2 \pi>2 \pi$. Итак, предположение было неверно и предложение 6 доказано.

ДОКАЗАТЕЛЬСТВо ПРЕДЛОЖЕНИЯ 8. 1) Предположим, что сушествуют такие эквивалентные отрезки $\omega_{j} \leqslant \omega_{l}$, что для некоторого $\widehat{x} \in \omega_{k}$ будет выполнено $p_{k j}(\widehat{x})>p_{k l}(\widehat{x})$. Согласно определению 5 последнее неравенство можно преобразовать следующим образом:

$$
\widehat{x}+i_{k j}(\widehat{x})-h(\widehat{x})>\widehat{x}+i_{k l}(\widehat{x})-h(\widehat{x}) .
$$

Отсюда получаем, что должно быть выполнено $i_{k j}(\widehat{x})>i_{k l}(\widehat{x})$, но мы знаем, что $i_{k j}(\widehat{x}) \in \omega_{j}, i_{k l}(\widehat{x}) \in \omega_{l}$ и $\omega_{j} \leqslant \omega_{l}$. Пришли к противоречию.

2) Заметим, что согласно определению базисного отрезка при $x \in \omega_{k} h^{\prime}(x)>0$. Кроме того, при $j=k_{1}, k_{3}, \ldots, k_{m} i_{k j}^{\prime}(x)<0$. Отсюда получаем, что при $x \in \omega_{k}$ $p_{k j}^{\prime}(x)=1+i_{k j}^{\prime}(x)-h^{\prime}(x)<1$. Если же $j=k_{2}, k_{4}, \ldots, k_{m-1}$, то при $x \in \omega_{j}$ $h^{\prime}(x)>0$, а следовательно, $i_{k j}^{\prime}(x)>0$. Значит, при $x \in \omega_{k} p_{k j}^{\prime}(x)=1+$ $i_{k j}^{\prime}(x)-h^{\prime}(x)>1-h^{\prime}(x)$. Воспользовавшись предположением (2), получаем утверждение 2) предложения 8.

Для доказательства пп. 3) и 4) заметим, что для любого $x \in \omega_{k}$ и любого $j$ должно выполняться равенство $p_{k j}(x)-i_{k j}(x)=x-h(x)$. Так как правая часть не зависит от номера $j$, получаем важное соотношение, вьполненное для всех $x \in \omega_{k}$,

$$
p_{k k_{1}}(x)-i_{k k_{1}}(x)=p_{k k_{2}}(x)-i_{k k_{2}}(x)=\cdots=p_{k k_{m}}(x)-i_{k k_{m}}(x)=x-h(x) \text {. (9) }
$$

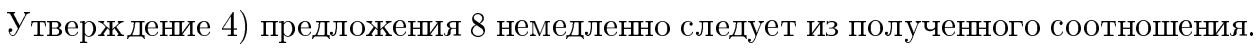
Для доказательства утверждения 3) продифференцируем равенство (9). При этом для $j=k_{1}, k_{3}, \ldots, k_{m}: i_{k j}^{\prime}(x)<0, p_{k j}^{\prime}(x)-i_{k j}^{\prime}(x)>p_{k j}^{\prime}(x)$, а при $j=k_{2}, k_{4}, \ldots$, $k_{m-1}: i_{k j}^{\prime}(x)>0, p_{k j}^{\prime}(x)-i_{k j}^{\prime}(x)<p_{k j}^{\prime}(x)$. Для того чтобы выполнялось равенство для производных при всех $j$, необходимо, чтобы функции $p_{k j}$ удовлетворяли 3 ). Предложение полностью доказано. 


\section{6. Теорема о реализации.}

ДокАЗАТЕЛЬство ТЕоремы 3. Зафиксируем базисньй отрезок $\omega_{k}$. Пусть отрезки разбиения $\omega_{k_{1}}, \omega_{k_{2}}, \ldots, \omega_{k_{m}}$ эквивалентны зафиксированному базисному отрезку. Построим на $\omega_{k}$ гладкую функцию $g_{k}(x)$ такую, что

$\forall x \in \omega_{k} \quad \max \left\{0, \max _{j=k_{1}, k_{3}, \ldots, k_{m}} p_{k j}^{\prime}(x)\right\}<g_{k}(x)<\min \left\{\min _{j=k_{2}, k_{4}, \ldots, k_{m-1}} p_{k j}^{\prime}(x), 1\right\}$

и со следующими граничными условиями:

1) если $x_{k}$ - точка разбиения между базисным и небазисным отрезками, то $g_{k}\left(x_{k}^{-}\right)=1$

2) если $x_{k}$ - точка разбиения между двумя базисньпи отрезками, то $0<$ $g_{k}\left(x_{k}^{-}\right)=g_{k+1}\left(x_{k}^{+}\right)<1$

$3)$ если же $x_{k-1}-$ точка разбиения между небазисным и базисным отрезками, то $0<g_{k}\left(x_{k-1}^{+}\right)<1$.

Кроме того, потребуем, чтобы

$$
\int_{x_{k-1}}^{x_{k}} g_{k}(y) d y=x_{k_{1}}-x_{k_{1}-1}+p_{k k_{1}}\left(x_{k}\right)-p_{k k_{1}}\left(x_{k-1}\right)=Q .
$$

Существование функции $g_{k}$ во внутренних точках базисного отрезка $\omega_{k}$ следует из неравенства (4), а достижимость граничных условий доказана ниже.

1) Справедливо следующее:

$$
\min _{j=k_{2}, k_{4}, \ldots, k_{m-1}} p_{k j}^{\prime}\left(x_{k}^{-}\right)>\max _{j=k_{1}, k_{3}, \ldots, k_{m}} p_{k j}^{\prime}\left(x_{k}^{-}\right) \geqslant p_{k k_{1}}^{\prime}\left(x_{k}^{-}\right) .
$$

Так как $x_{k}$ - точка разбиения между базисным отрезком $\omega_{k}$ и небазисным $\omega_{k+1}$, то эти отрезки эквивалентны, а следовательно, $p_{k k_{1}}^{\prime}\left(x_{k}^{-}\right)=p_{k k+1}^{\prime}\left(x_{k}^{-}\right)=1$.

2) Пусть $\omega_{k}$ и $\omega_{k+1}$ базисные отрезки. Для удобства вместо $\omega_{k+1}$ будем писать $\omega_{\mu}$. Знаем, что $\omega_{k} \cap \omega_{\mu}=x_{k}$, и хотим доказать, что $g_{k}\left(x_{k}^{+}\right)=g_{\mu}\left(x_{k}^{-}\right)$. Для этого достаточно показать, что верны следуюшие неравенства:

$$
\begin{aligned}
& \max _{j=\mu_{1}, \mu_{3}, \ldots, \mu_{\lambda}} p_{\mu j}^{\prime}\left(x_{k}^{-}\right) \leqslant \min _{j=k_{2}, k_{4}, \ldots, k_{m-1}} p_{k j}^{\prime}\left(x_{k}^{+}\right), \\
& \max _{j=k_{1}, k_{3}, \ldots, k_{m}} p_{k j}^{\prime}\left(x_{k}^{+}\right) \leqslant \min _{j=\mu_{2}, \mu_{4}, \ldots, \mu_{\lambda-1}} p_{\mu j}^{\prime}\left(x_{k}^{-}\right) .
\end{aligned}
$$

Докажем первое из этих неравенств:

$$
\begin{gathered}
p_{k k_{1}}^{\prime}\left(x_{k}^{+}\right)-i_{k k_{1}}^{\prime}\left(x_{k}^{+}\right)=\cdots=p_{k k_{m}}^{\prime}\left(x_{k}^{+}\right)-i_{k k_{m}}^{\prime}\left(x_{k}^{+}\right)=1-h^{\prime}\left(x_{k}^{+}\right) \\
p_{\mu \mu_{1}}^{\prime}\left(x_{k}^{-}\right)-i_{\mu \mu_{1}}^{\prime}\left(x_{k}^{-}\right)=\cdots=p_{\mu \mu_{\lambda}}^{\prime}\left(x_{k}^{-}\right)-i_{\mu \mu_{\lambda}}^{\prime}\left(x_{k}^{-}\right)=1-h^{\prime}\left(x_{k}^{-}\right) .
\end{gathered}
$$

Так как $h^{\prime}\left(x_{k}^{+}\right)=h^{\prime}\left(x_{k}^{-}\right)$, то $\forall j, l \quad p_{\mu j}^{\prime}\left(x_{k}^{-}\right)-i_{\mu j}^{\prime}\left(x_{k}^{-}\right)=p_{k l}^{\prime}\left(x_{k}^{+}\right)-i_{k l}^{\prime}\left(x_{k}^{+}\right)$. Более того,

$$
\begin{gathered}
\forall j=\mu_{2}, \mu_{4}, \ldots, \mu_{\lambda-1} \quad i_{\mu j}^{\prime}\left(x_{k}^{-}\right) \geqslant 0, \\
\forall l=k_{1}, k_{3}, \ldots, k_{m} \quad i_{k l}^{\prime}\left(x_{k}^{+}\right) \leqslant 0, \\
p_{k l}^{\prime}\left(x_{k}^{+}\right) \leqslant p_{k l}^{\prime}\left(x_{k}^{+}\right)-i_{k l}^{\prime}\left(x_{k}^{+}\right)=p_{\mu j}^{\prime}\left(x_{k}^{-}\right)-i_{\mu j}^{\prime}\left(x_{k}^{-}\right) \leqslant p_{\mu j}^{\prime}\left(x_{k}^{-}\right) .
\end{gathered}
$$


Второе неравенство доказьвается аналогично.

3) Немедленно следует из неравенства (4).

Осталось показать, что можно добиться выполнения (11). Из условий (5), (6) следует, что $Q=x_{k}-h\left(x_{k}\right)-\left(x_{k-1}\right)-h\left(x_{k-1}\right)$, а это означает, что $0<Q<$ $x_{k}-x_{k-1}$. Остается заметить, что $0<g_{k}(x)<1$ и интегрирование ведется по базисному отрезку $\omega_{k}$.

Итак, функция $g_{k}$ построена.

Далее на базисном отрезке $\omega_{k}$ построим функцию

$$
i_{k j}^{\prime}(x)=p_{k j}^{\prime}(x)-g_{k}(x)
$$

Легко заметить, что в силу неравенств (10) при $j=k_{1}, k_{3}, \ldots, k_{m}$ имеем $i_{k j}^{\prime}(x)<0$, а при $j=k_{2}, k_{4}, \ldots, k_{m-1} i_{k j}^{\prime}(x)>0$. Проинтегрировав равенство $(13)$, получаем

$$
i_{k j}(x)=p_{k j}(x)-\int_{x_{k-1}}^{x} g_{k}(y) d y+c .
$$

Константа $c$ выбирается так, чтобы выполнялись граничные условия

$$
i_{k k_{1}}\left(x_{k-1}\right)=x_{k_{1}}, \quad i_{k k_{1}}\left(x_{k}\right)=x_{k_{1}-1}
$$

(в силу равенств $(5),(6)$ граничные условия для $i_{k k_{2}}, \ldots, i_{k k_{m}}$ также будут выполнены). Из первого условия находим, что $c=x_{k_{1}}-p_{k k_{1}}\left(x_{k-1}\right)$, при этом второе условие будет вьполнено в силу условия (11).

Искомая функция $h$ теперь может быть построена на отрезке [0;2 $]$. На базисном отрезке $\omega_{k}$ функция $h$ задается равенством $h(x)=x+i_{k j}(x)-p_{k j}(x)$. Для построения функции $h$ на небазисном отрезке $\omega_{l}$ рассмотрим базисный отрезок $\omega_{K}$, эквивалентный $\omega_{l}$. Тогда $h(x)=h\left(i_{K l}^{-1}(x)\right), x \in \omega_{l}$. Правая часть равенства нам известна, следовательно, мы построили функцию $h$ на всех отрезках разбиения. Осталось проверить, что построенная таким образом функция $h$ удовлетворяет требованиям типичности и $h^{\prime}(x)<1$.

Требования типичности 1), 3) и 4) выполнены в силу выбора функций $p_{k j}$ и построения функции $h$. Осталось показать, что построенная функция $h$ имеет $n+1$ экстремум на интервале $[0 ; 2 \pi)$ и является морсовской. Для этого решим уравнение $h^{\prime}(x)=0$. На базисном отрезке $\omega_{k}$ верно следуюшее преобразование:

$$
h^{\prime}(x)=1+i_{k j}^{\prime}(x)-p_{k j}^{\prime}(x) \stackrel{(13)}{=} 1-g_{k}(x) \stackrel{(10)}{\neq} 0 .
$$

Пусть на небазисном отрезке $\omega_{l}$ сушествует точка $x_{0}$ такая, что $h^{\prime}\left(x_{0}\right)=0$. Согласно формуле, задающей функцию $h$ на небазисном отрезке,

$$
h^{\prime}\left(x_{0}\right)=h^{\prime}\left(i_{k l}^{-1}\left(x_{0}\right)\right)\left(i_{k l}^{-1}\left(x_{0}\right)\right)^{\prime}=0 .
$$

Как показано выше, производная функции $h$ не обрашается в нуль на базисном отрезке, а следовательно, должно выполняться следующее равенство: $\left(i_{k l}^{-1}\left(x_{0}\right)\right)^{\prime}=0$. По теореме об обратной функции это равенство преобразуется в следующее:

$$
\frac{1}{i_{k l}^{\prime}\left(y_{0}\right)}=0, \quad y_{0} \in \omega_{k}
$$


Откуда в силу (13) получаем $1 /\left(p_{k l}^{\prime}\left(y_{0}\right)-g_{k}\left(y_{0}\right)\right)=0$, а это противоречит ограниченности $p_{k l}^{\prime}\left(y_{0}\right)-g_{k}\left(y_{0}\right)$. Итак, предположение о существовании точки $x_{0}$ было неверньм.

Из вышесказанного следует, что функция $h$ может иметь экстремумы только в точках разбиения $\Omega$. Зафиксируем точку разбиения $x_{s}$ и рассмотрим все возможные случаи расположения отрезков разбиения.

а) Отрезок $\omega_{s}$ базисный, $\omega_{s+1}$ - нет. В этом случае согласно граничному условию, наложенному на функцию $g_{s}$ при построении, $g_{s}\left(x_{s}^{-}\right)=1$, следовательно, в силу гладкости функции $g_{s} h^{\prime}\left(x_{s}\right)=1-g_{s}\left(x_{s}\right)=0$. Итак, точка разбиения $x_{s}$, слева от которой находится базисный отрезок, а справа небазисный, является точкой локального экстремума функции $h$.

b) Отрезки $\omega_{s}$ и $\omega_{s+1}$ базисные. Из построения $g_{s}$ следует, что $h^{\prime}\left(x_{s}^{-}\right)=$ $h^{\prime}\left(x_{s}^{+}\right)$и $h^{\prime}\left(x_{s}\right) \neq 0$, так как иначе второй отрезок не может быть базисным.

c) Отрезок $\omega_{s}$ небазисный, a $\omega_{s+1}$ базисный. Рассуждения аналогичны предыдущему случаю.

d) Отрезки $\omega_{s}$ и $\omega_{s+1}$ небазисные. Этот случай разобьем на два:

i) рассматриваемые отрезки принадлежат одному классу эквивалентности с базисньм отрезком $\omega_{k}$, тогда

$$
\begin{gathered}
h^{\prime}\left(x_{s}^{-}\right)=\left(h\left(i_{k s}^{-1}\left(x_{s}^{-}\right)\right)\right)^{\prime}=h^{\prime}\left(i_{k s}^{-1}\left(x_{s}^{-}\right)\right)\left(i_{k s}^{-1}\left(x_{s}^{-}\right)\right)^{\prime}, \\
h^{\prime}\left(x_{s}^{+}\right)=\left(h\left(i_{k s+1}^{-1}\left(x_{s}^{+}\right)\right)\right)^{\prime}=h^{\prime}\left(i_{k s+1}^{-1}\left(x_{s}^{+}\right)\right)\left(i_{k s+1}^{-1}\left(x_{s}^{+}\right)\right)^{\prime} ;
\end{gathered}
$$

так как $\left(i_{k s}^{-1}\left(x_{s}^{-}\right)\right)^{\prime}=\left(i_{k s+1}^{-1}\left(x_{s}^{+}\right)\right)^{\prime}=0$, в данном случае точка разбиения является локальным экстремумом функции $h$;

ii) $\omega_{s}$ принадлежит классу эквивалентности с базисным отрезком $\omega_{k}$, a $\omega_{s+1}$ другому классу с базисньм отрезком $\omega_{k+1}$, тогда

$$
\begin{gathered}
h^{\prime}\left(x_{s}^{-}\right)=\left(h\left(i_{k s}^{-1}\left(x_{s}^{-}\right)\right)\right)^{\prime}=\frac{h^{\prime}\left(i_{k s}^{-1}\left(x_{s}^{-}\right)\right)}{i_{k s}^{\prime}\left(x_{k}^{-}\right)}, \\
h^{\prime}\left(x_{s}^{+}\right)=\left(h\left(i_{k+1 s+1}^{-1}\left(x_{s}^{+}\right)\right)\right)^{\prime}=\frac{h^{\prime}\left(i_{k+1 s+1}^{-1}\left(x_{s}^{+}\right)\right)}{i_{k+1 s+1}^{\prime}\left(x_{k}^{+}\right)} ;
\end{gathered}
$$

отсюда получаем, что в данном случае $h^{\prime}\left(x_{s}^{-}\right)=h^{\prime}\left(x_{s}^{+}\right) \neq 0$.

Покажем, что для построенной функции $h$ выполнено условие (2).

На базисном отрезке $\omega_{k}$ имеем $h^{\prime}(x)=1+i_{k j}^{\prime}(x)-p_{k j}^{\prime}(x)=1-g_{k}(x)$. В силу (10) $0<g_{k}(x)<1$, а следовательно, для $x \in \omega_{k}$ выполнено $h^{\prime}(x)<1$.

Проверим справедливость этого условия на небазисном отрезке $\omega_{l}$, эквивалентном базисному отрезку $\omega_{k}$. По построению при $x \in \omega_{l}$ имеем

$$
h^{\prime}(x)=h^{\prime}\left(i_{k l}^{-1}(x)\right)\left(i_{k l}^{-1}(x)\right)^{\prime}=\frac{h^{\prime}\left(i_{k l}^{-1}(x)\right)}{i_{k l}^{\prime}\left(i_{k l}^{-1}(x)\right)} .
$$

Рассмотрим точку $z$, принадлежащую базисному отрезку $\omega_{k}, z=i_{k l}^{-1}(x)$, и покажем, что $h^{\prime}(z) / i_{k l}^{\prime}(z)<1$. Из вышесказанного мы знаем, что на базисном отрезке $0<h^{\prime}(z)<1$. 
Если $i_{k l}^{\prime}(z)<0$, то неравенство выполнено.

Предположим, что $i_{k l}^{\prime}(z)>0$. По построению функции $h$ имеем $h^{\prime}(z)=1+$ $i_{k j}^{\prime}(z)-p_{k j}^{\prime}(z)$, откуда $i_{k j}^{\prime}(z)-h^{\prime}(z)=p_{k j}^{\prime}(z)-1$. В силу второго утверждения предложения $8 p_{k j}^{\prime}(z)-1>0$. Итак, неравенство $h^{\prime}(z) / i_{k j}^{\prime}(z)<1$ доказано, а из него вытекает, что $h^{\prime}(x)<1 \forall x \in \omega_{l}$.

Теорема полностью доказана.

\section{§3. Случай добавочной функции, зависящей от параметра}

ПРЕДЛОЖЕНИЕ 13. Пусть семейство функиий $h_{a}$ сходится $x$ функиии $h$ по норме пространства $C^{3}$ при а $\rightarrow \infty$. Тогда разбиения $\Omega_{a}$ и $\Omega$, порожсденные функциями $h_{a}$ и $h$ соответственно, әквивалентны, т.е. имеют одинаковые характеристические графы.

ДоКАЗАТЕЛЬСТво. Из сходимости следует, что начиная с некоторого $a$ функции $h_{a}$ и $h$ имеют одинаковые updown подстановки. Как отмечалось ранее, updown подстановка однозначно определяет характеристический граф. Что и требовалось доказать.

Разбиение $\Omega$ определено таким образом, что на двух фиксированных эквивалентных отрезках разбиения задача описания циклов периода два сводится к унимодальному случаю. Следовательно, при фиксированной паре номеров $(k, j)$ применимы утверждения из $[7 ; \S 2.6]$. Сформулируем эти утверждения для случая произвольной функции $h$.

ПРЕДЛОЖЕНИЕ 14. Пусть семейство морсовских функиий $h_{a}(x)$ сходится $\kappa$ морсовской функции $h$ по норме пространства $C^{3}$ при $a \rightarrow \infty$. Рассмотрим набор функций $\left\{i_{k j}(x, a)\right\}$, определенных равенством $h_{a}\left(i_{k j}(x, a)\right)=h_{a}(x), a$ также набор функиий $\left\{i_{k j}(x)\right\}$, заданный равенством $h\left(i_{k j}(x)\right)=h(x)$. Тогда каждой функиии из первого набора соответствует единственная функция второго набора и наоборот. Более того, при фиксированных $(k, j)$ функция $i_{k j}(x, a)$ сходится $к$ функции $i_{k j}(x)$ по норме пространства $C^{2}$ на кажсдом отрезке, лежащем строго внутри $\omega_{k}$ при $a \rightarrow \infty$.

ПреДЛОЖенИЕ 15. Рассмотрим семейство

$$
\widetilde{f}_{a}: x \mapsto-x+a+h_{a}(x), \quad a \in[2 \pi m ; 2 \pi(m+1)],
$$

и разбиение $\Omega_{a}$, которое в силу предложения 13 эквивалентно разбиению $\Omega$ при любом значении параметра. Пусть $\left\{i_{k j}(x, a)\right\}$ - набор инволютивных функиий, где номера $(k, j)$ пробегают номера всех пар әквивалентных отрезков. Тогда множество пар $(x, a)$, где $x$ - точка 2-цикла семейства $\widetilde{f}_{a}$, совпадает с множсеством пар $(x, a)$, являюшихся решениями уравнения

$$
a=x+i_{k j}(x, a)-h_{a}(x)+2 \pi m .
$$

Кроме того, для фиксированных $(k, j)$ при достаточно больших значениях $m$ уравнение (16) имеет единственное решение $a=p_{k j}^{m}(x)$ и при этом решение принадлежит классу $C^{2}$. 
ПРЕДЛОЖЕНИЕ 16. Пусть $\left\{p_{k j}^{m}(x)\right\}$ - набор функций циклов семейства (15) при достаточно больиих значениях $m$. Семейство $\left\{h_{a}\right\}$ сходится $к$ морсовской функции $h$ по норме $C^{3}$ при $а \rightarrow \infty$. Набор $\left\{p_{k j}(x)\right\}$ является набором функций ииклов семейства $f_{a}: x \mapsto-x+a+h(x)$. Зафиксируем номера $k, j$ эквивалентных отрезков разбиения $\Omega$. Тогда семейство $p_{k j}^{m}-2 \pi m$ сходится $\kappa$ функции $p_{k j}$ по норме пространства $C^{2}$ при $m \rightarrow \infty$.

Теорема 4. Рассмотрим семейство диффеоморфизмов $\widetilde{f}_{a}: x \mapsto-x+a+$ $h_{a}(x)$, где семейство функций $h_{a}$ сходится $\kappa$ функции $h$ по норме $C^{3}$ при $a \rightarrow \infty$, и семейство $f_{a}: x \mapsto-x+a+h(x)$. Тогда при значениях параметра $а \in[2 \pi m ; 2 \pi(m+1)]$ семейство $\widetilde{f}_{а}$ начиная с некоторого достаточно больиого $m$ имеет бифуркационный сценарий, эквивалентный в смысле определения 9 бифуркационному сиенарию семейства $f_{a}$ при $a \in[0 ; 2 \pi]$.

\section{Список литературы}

1. Ильяиенко Ю. С., Ли Вейгу. Нелокальные бифуркации. М.: МЦНМО ЧеРо, 1999.

2. Афраймович В. С., ШІильников Л. П. О некоторых глобалњных бифуркациях, связанных с исчезновением неподвижной точки типа седло-узел // Докл. АН СССР. 1974. T. 219. № 3. C. 1281-1285.

3. Арнольд В.И., Афраймович В.С., Ильяиенко Ю.С., Шильников Л.П. Теория бифуркаций // Итоги науки и техники. Совр. проблемы матем. Фундамент. направления. Динамические системы. Т. 5. М.: ВИНИТИ, 1986. С. 5-218.

4. Hirsch V.W., Pugh C.C., Shub M. Invariant manifolds. New York: Springer-Verlag, 1977. (Lecture Notes in Math. V. 583.)

5. Fenichel $N$. Persistence and smoothness of invariant manifolds for flows // Indiana Univ. Math. J. 1971. V. 21. P. 193-226.

6. Медведев В.С. О новом типе бифуркаций на многообразиях // Матем. сб. 1980. T. 113 (155). №3. C. 487-492.

7. Борисюк A. Р. Глобальные бифуркации на бутылке Клейна. Унимодальный случай // Матем. заметки. 2002. Т. 71. № 3. С. 348-363.

8. Арнольд В.И., Варченко А.Н., Гусейн-Заде С. М. Особенности дифференцируемых отображений. I. М.: Наука, 1982. 